\title{
An approach to determine the coronal plane deformity of the distal tibia intraoperatively in the treatment of tibial fracture with intramedullary nail fixation
}

\section{Yao Lu}

Department of Orthopedics Truama, Xi'an Honghui Hospital,School of Medicine Xi'an Jiaotong University

\section{Jie Yang}

Department of Orthopedics Surgery II,Langfang people's hospital

Yibo Xu ( $D$ moria_33@163.com )

Department of Traumatology and Orthopedics, Xi'an Honghui Hospital, College of Medicine Xi'an Jiaotong University

\section{Teng Ma}

Department of Orthopedics Trauma,Xi'an Honghui Hospital,School of Medicine Xi'an Jiaotong University

\section{Ming Li}

Department of Orthopedics Trauma, Xi'an Honghui Hospital,School of Medicine Xi'an Jiaotong University

\section{Cheng Ren}

Department of Orthopedics Trauma,Xi'an Honghui Hospital,School of Medicine Xi'an Jiaotong University

\section{Qian Wang}

Department of Orthopedics Trauma,Xi'an Honghui Hospital,School of Medicine Xi'an Jiaotong University

\section{Zhong Li}

Department of Orthopedics Trauma,Xi'an Honghui Hospital,School of Medicine Xi'an Jiaotong University

\section{Kun Zhang}

Department of Orthopedics Trauma,Xi'an Honghui Hospital,School of Medicine Xi'an Jiaotong University

\section{Research article}

Keywords: tibia fractures; intramedullary nailing; internal fixation; valgus; varus 
Posted Date: August 18th, 2020

DOl: https://doi.org/10.21203/rs.3.rs-51792/v1

License: (c) (i) This work is licensed under a Creative Commons Attribution 4.0 International License. Read Full License 


\section{Abstract}

Background To develop a new approach to determine the existence of coronal plane deformity (valgus and varus) intraoperatively in the treatment of tibial fracture with closed reduction and intramedullary nail fixation.

Methods A retrospective analysis was conducted by enrolling 33 consecutive patients with tibial fracture who underwent closed reduction and intramedullary nail fixation from January 2018 to January 2019 in our trauma center. There were 23 males and 10 females aged from 22 to 69 years old with an average of 41.3. The standard anteroposterior and lateral preoperative radiographs were performed routinely. After insertion of the tibial intramedullary nail through the standard entry point intraoperatively, the parallel relationship between the distal horizontal interlocking screw and the tibiotalar joint surface on the anteroposterior fluoroscopy was used to determine the occurrence of valgus or varus deformity of the distal tibial fragment.

Results 33 patients were postoperatively followed up for 13 to 25 months (mean 18.7 months). The fractures achieved bone union in 4.3 months by average (ranging from 3 to 6 months). The lateral distal tibial angle (LDTA) of the unaffected side which is measured postoperatively was $87.3^{\circ}-89.6^{\circ}$ (average $88.7^{\circ} \pm 0.8^{\circ}$ ), and the LDTA of the affected side was $87.5^{\circ}-90.4^{\circ}$ (average $88.9^{\circ} \pm 1.1^{\circ}$ ). There was no statistically significant difference between the unaffected side and the affected side $(t=-1.865, p=0.068)$. The intraoperative measurement indicated 6 cases of valgus angulation and 3 cases of varus angulation deformities, and all the deformities were corrected accordingly during the surgery. According to the Olerud-Molander Ankle Score, the clinical outcomes demonstrated 22 excellent cases, 8 good cases, 2 fair cases, and 1 poor case of all the fractures at 12 months after surgery.

Conclusion During closed reduction and intramedullary nail fixation for tibial fractures, the parallel relationship between the distal horizontal interlocking screw and the tibiotalar joint surface on the intraoperative anteroposterior films showed the better discriminative ability to determine the onset of valgus or varus deformity of the distal tibial fragment after the tibial intramedullary nail inserted through the standard entry point.

Level of Evidence: Level IV, case series study.

\section{Introduction}

Tibial fractures are common types of fractures in traumatology orthopedics, most of which are highenergy injuries and always accompanied by soft tissue injuries[1]. Based on the unique blood supply and anatomical characteristics, soft tissue concerns with plate fixation including infections, delayed union or nonunion of the fracture and implants associated issues resulted in the increased application of intramedullary nail fixation, which obtains the extra benefit of being a load sharing device to allow early weight bearing[2-4]. Because the tibial intramedullary nail fixation has little interference with the soft tissue around the fracture site and shows a high union rate for the fractures, it has become the standard 
treatment of choice for displaced tibial fractures in adults [5-7]. However, due to the inability to view the fractured site directly and film the entire tibia with intraoperative C-arm fluoroscopy when performing closed reduction and tibial intramedullary nail fixation, it is a substantial challenge to determine whether the distal tibia valgus or varus deformity occurs during the surgery [8]. In this study, we retrospectively analyzed the eligible data collected from 33 patients with comminuted tibial fractures treated with tibial intramedullary nails from January 2018 to January 2019, and used the parallel relationship between the distal horizontal interlocking screw and the tibiotalar joint surface on the anteroposterior fluoroscopy film to determine the onset of valgus or varus deformity of the distal tibial fragment of the tibial fractures.

\section{Material And Methods}

\section{Inclusion and exclusion criteria}

Inclusion criteria: 1 ) patients aged over 18 years old; 2) Gustilo-Anderson type $\nabla$ and $\otimes$ open tibia fracture; 3) AO/OTA classification: type 42-B3 and type C closed tibia fracture; 4) no existed deformity of knee, ankle joints or tibia was discovered before injury. Exclusion criteria: 1) Gustilo-Anderson type $₫$ open tibia fracture; 2) old tibia fracture; 3 ) simple tibia fracture, 42-A, B1 and B2 types according to the AO/OTA classification; 4) the onset of serious knee and ankle joint diseases such as rheumatoid arthritis and gouty arthritis; 5) Previous tibia fracture and deformity union; 6) Patients who refused surgery.

\section{Research Design}

A single-center, retrospective study was conducted at our trauma center from January 2018 to January 2019. All eligible data was collected and reviewed based on the clinical material database including age, gender, mechanism of injury, AO/OTA classification and Gustilo-Anderson classification[9], the average interval from injury to surgery, the lateral distal tibial angle (LDTA) of the unaffected and affected side, complications and the Olerud-Molander Ankle Score.

\section{The approach to determine the onset of valgus or varus deformity of the distal tibial fragment}

The C-arm fluoroscopy films were used to obtain standard anteroposterior view. The tibial intramedullary nail was projected as a vertical line (line a) on the coronal plane, therefore, when the intramedullary nail was inserted through the standard entry point, line a would be perpendicular to the projection line of the knee joint surface (line b) on the coronal plane(Fig. 1). Theoretically, when the distal tibia does not combine with the concomitant valgus or varus deformity, line a would also be perpendicular to the projection line of the tibiotalar joint plane (line $\mathrm{c}$ ) on the coronal plane; meanwhile the projection line of the distal horizontal interlocking screw axis(line d) on the coronal plane is designed to be perpendicular to intramedullary nail(line a). Based on the geometrical theory that if a straight line is vertically intersected by multiple straight lines in the same plane, then these intersected lines must be parallel with each other. Theoretically, the three lines of the knee joint plane, the distal horizontal interlocking screw and the tibiotalar joint surface projected on the coronal plane should be parallel (that is line b, $c$ and $d$ are parallel to each other and are perpendicular to the line a simultaneously). Moreover the distal interlocking screw and the tibial intramedullary nail are both made of metal materials[10], the probability to generate 
deviation in the locking process is very low (line $d$ and line a were designed to be perpendicular to each other), therefore, if an included angle emerged between the projection lines of the distal horizontal interlocking screw and the tibiotalar joint plane on the coronal plane (i.e. the line $d$ and the line $c$ are not parallel to each other) (Fig. 3), it is demonstrated that the valgus or varus deformity occurs at the fracture of the distal tibia in the coronal plane. Meanwhile the fibula can be used as a reference. If the included angle points to the fibular side, the valgus deformity exists, and if the angle faces away from the fibula, then varus deformity exists.

\section{Surgical Procedure}

Before the operation, the anteroposterior and lateral view for the entire tibia were performed to determine the severity degree, location and displacement of the fracture fragments. CT scans were conducted to identify the injury of joint fractures for the patients whose fracture lines affect the knee or ankle joint surface. The patient underwent epidural anesthesia or general anesthesia in the supine position on the radiolucent orthopedic surgical table. The tourniquet was ligated to the proximal thigh after anesthesia, and the hip on the affected side was raised. Open fractures were irrigated and debride. With respect to the fibula fractures and fracture line located within $8 \mathrm{~cm}$ above the ankle mortise, firstly we fixed the lateral malleolus through a lateral approach and careful dissection was performed to avoid superficial peroneal nerve injury. After reduction of the distal fibula into the tibial incusura, the $3.5 \mathrm{~mm}$ fibular locking compression plate was performed before confirming by fluoroscopy, then the full layer was temporarily sutured to maintain skin tension. A standard anterior patellar approach was adopted for nail insertion, the patellar tendon was split and the fat pad of subpatellar was cleaned to expose the tibial plateau slope. The nail starting point must be determined with fluoroscopy guidance before opening the medullary cavity, which is especially important. The ideal entry point should be just medial to the lateral tibia spine on the AP fluoroscopic view and just anterior to the articular surface on the lateral view [11] (Fig. 2). After preparation of the entry portal by awl with the knee hyperflexed, longitudinal traction and appropriately directed forces were manually applied to the limb to obtain provisional alignment and fulfill fracture reduction. The finger reduction device was inserted and the $\mathrm{C}$-arm fluoroscopy was used to examine the fracture alignment condition and the depth and position of finger reduction device in the distal medullary cavity. The finger reduction device was removed once it was placed at the right position and replaced with a guide wire. The fracture reduction was maintained by the assistant and the appropriate length and diameter intramedullary nail was inserted along the guide wire after gradual reaming. The tip of the intramedullary nail was placed as close as possible to the articular surface of the distal tibia. Two locking screw were locked into the horizontal plane of the distal tibia. $\mathrm{C}$ arm fluoroscopic images were used to confirm if there was an included angle existed between the axis of the distal horizontal interlocking screw and the horizontal line of the tibiotalar articular surface on the AP film. When the two lines were parallel and had no intersection, two distal screws could be locked. If there existed an included angle, the direction of this angulation had to be identified first, then remove the interlocking screws and the intramedullary nail; the blocking screw was placed at the anteroposterior direction near the included angle on the side of the distal fracture fragment, and the intramedullary nail was inserted along the guide pins after another reaming process, and 1 to 2 locking screws were relocked with the nail. The C-arm X-ray 
machine continued to monitor whether the included angle between the axis of the distal horizontal interlocking screw and the horizontal line of the tibiotalar articular surface on the AP view corrected. When such an angle disappeared, the proximal screws could be locked; whereas when the included angle was still present, the procedures had to be repeated until the included angle corrected (Fig. 3). After the proximal screws were locked, the tail cap was installed. Once we achieved a satisfactory reduction on the fluoroscopy, the irrigation and suction drainage were performed and then the incision was closed sequentially

\section{Postoperative Management}

The affected limb was raised and routine second-generation cephalosporin antibiotics were applied for 24 hours postoperatively in order to prevent infection. On the second day after the operation, the postoperative $\mathrm{X}$-ray films were performed and the patient was encouraged to take active and passive functional exercises for the affected knee, ankle, and toes, and CPM machine-assisted exercise if necessary. The thrombolytics or anticoagulant therapy were given after surgery to prevent deep vein thrombosis of the lower extremities.

The both side full-length lower limbs anteroposterior and lateral X-ray films were taken after removal of the drainage tube postoperatively to investigate the reduction and implant location, meanwhile to measure the LDTA on both affected and contralateral sides. Thus could determine whether the fracture fragment of distal tibia combined valgus or varus deformity.

The application of LDTA to estimate the existed deformity in the coronal plane of the fracture according to Paley's deformity analysis method [12]. It is considered as distal tibia valgus deformity presented when the LDTA is $<86$ degrees and the varus deformity for LDTA $>92$ degrees. Compared to the unaffected ankle joint, an included angle of more than 10 degrees of varus deformity or 15 degrees valgus deformity requires reoperation. Follow-up took place once a month for the first 3 months after surgery, then extended to every 3 to 6 months after first 3 follow-ups of the surgery, and finally every 6 to 12 months after 1 year of surgery.

\section{Statistical analysis}

Statistical analysis was performed by SPSS 24.0 statistical software (IBM, USA). A Shapiro-Wilk test for normality was conducted for all continuous data, and the continuous data with the normal distribution was described in the form of mean \pm standard deviation whereas the categorical data was described in number of cases (percentage). The continuous data with the normal distribution such as the LDTA between the affected side and contralateral unaffected side were analyzed by Student's t-test and the Mann-Whitney U-test for those not with the normal distribution. As for the categorical variables, the $\chi 2$ test was performed. $p<0.05$ was selected as the threshold for statistically significant.

\section{Results}


We enrolled 33 consecutive patients who were postoperatively followed up for 13 to 25 months (mean 18.7 months). There were 23 males and 10 females aged from 22 to 69 years old with an average of 41.3. Of the mechanism of injury, 26 cases resulted from road traffic accident and 7 cases from accidental falling from standing height. Based on the AO/OTA classification, specificly there were 7 cases of type B3, 4 cases of $\mathrm{C} 1,10$ cases of $\mathrm{C} 2$, and 12 cases of C3. Of the 5 open fractures, there were 3 Gustilo type I and 2 type II cases. The average interval from injury to surgery was 4.3 days (2 to 11 days). The fractures achieved bone union in 4.3 months by average (ranging from 3 to 6 months). The lateral distal tibial angle (LDTA) of the unaffected side which is measured postoperatively was $87.3^{\circ}-89.6^{\circ}$ (average $88.7^{\circ} \pm 0.8^{\circ}$ ), and the LDTA of the affected side was $87.5^{\circ}-90.4^{\circ}$ (average $88.9^{\circ} \pm 1.1^{\circ}$ ). There was no statistically significant difference between the unaffected side and the affected side $(t=-1.865, p=$ 0.068).

The measure of intraoperative included angle demonstrated 24 cases of normal, 6 cases of valgus angulation and 3 cases of varus angulation. All the valgus or varus deformities were corrected during the surgery.

The total complication rate of our study was $60.6 \%$ (20 cases) including 4 cases of deep vein thrombosis, 1 case of infection and delayed union, we also noticed that there were 15 cases of slight to moderate anterior knee pain which may be one of the most frequently discussed complications at the last follow up. According to the Olerud-Molander Ankle Score [13], the clinical outcomes indicated that 22 cases were excellent, 8 cases were good, 2 cases were normal, and 1 case was poor at 12 months after the surgery. The excellent and good rate was $90.9 \%$. One case with poor efficacy belonged to AO/OTA type C3 and Gustilo-Anderson II open fracture. The tibial intramedullary nail fixation was performed 1 week after the debridement and external fixation. Of this patient, infection occurred 5 days after the nailing fixation. Debridement procedure was performed again and internal fixation were replaced by external fixation simultaneously. The ankle joint function of this patient showed poor outcomes after infection control at late stage.

\section{Discussions}

The anterior medial tibia is covered by little and thin soft tissues, which is more obviously presented at the distal end. Fractures often occurred with concomitant local skin contusion and soft tissue injuries. In addition, blood supply to the distal tibia is generally poor and the extraosseous blood supply diminished under the fracture condition. Meanwhile reaming would eliminate the remain blood supply which may predispose the fracture to infection, delayed union and nonunion. Therefore, it is particularly crucial to choose an optimal internal fixation method that provides stable fixation and less interference with soft tissue simultaneously, thus can promote the recovery of limb soft tissue and fracture healing. External fixation is considered to be an effective fixation method. However, Ristiniemi et al[14]recently found that external fixation shows more complications when applied for the treatment of tibial fractures including nail trajectory infections, ankle stiffness and delayed fracture union. The authors treat 47 tibial fractures with combined external fixation, which brought the sequelae of delayed union for $25.5 \%$ of the patients. 
The advantages of applying open reduction and internal fixation were that the fracture site could be exposed sufficiently and the reduction could be fulfilled anatomically under direct vision. Researchers [3, 15-17] reported that the deformed union rate of fractures after plate fixation was less than $10 \%$, but the risk of postoperative complications with soft tissues was higher, including superficial infections, deep infections, delayed wound healing, and necrosis of soft tissues. In the early 1940 s, the intramedullary nail system was introduced to treat tibial shaft fractures, which can reduce the deformity union for tibial fracture and decrease the formation of pseudo-articular joints after the surgery, meanwhile promote the ossification of fractured callus. Moreover, the intramedullary nails have little interference with the soft tissue around the fracture site. Previous studies [18] noted that intramedullary nails have a shorter operative time in the treatment of tibial fractures and better postoperative function recovery. Therefore, in this study, we selected closed reduction and intramedullary nail fixation for tibial fractures. Currently, intramedullary nailing has become the popular treatment strategy for diaphyseal fractures of the tibia[5, 19]. We noticed the occurrence of angulation deformity for the distal fracture fragment during the nail insertion, it also has been reported that even a small amount of residual alters load through the knee and ankle joint [20], and the alternation in force could attribute a predisposition to osteoarthritis [21, 22]. Hence correctly estimating the occurrence of angulation deformity intraoperatively is the essential prerequisite to better clinical outcomes for the patients.

\section{The reasons for the occurrence of distal tibia fragment valgus or varus deformity during the surgery}

We assume the reasons for the valgus and varus deformity were complicated, mostly resulted from losing the balance of forces around the fracture site during inserting the nail. In the treatment of tibial fractures with open reduction and internal fixation, owing to the sufficiently exposed fracture site and reduction under direct vision, varus or valgus deformity of the distal fragment is hardly to occur unless there were existed varus and valgus deformities before the injury. However, when comes to closed reduction and intramedullary nail fixation, the reduction conditions of the fractures cannot be examined under direct vision since the fracture site would not be exposed. Moreover, because the full-length tibial imaging is currently not available during the surgery in most case scenarios, poor reduction including varus and valgus cannot be avoided. Based on the anatomical characteristics of the distal tibia, the compartment muscles and tendons formed by the extensor and flexor muscles passes through the anterior and posterior tibia, the lateral side is the fibula while the medial side has no muscle groups. This may result in a low probability of sagittal plane deformity of distal frament such as apex anterior after the traction and reduction due to the ligamentotaxis and blockage of anterior and posterior hinges. However, varus or valgus deformity is prone to occur due to the lack of soft tissue hinges on the medial and lateral sides which were not able to neutralize the forces. 1) For middle tibial shaft fractures, if the isthmus remains intact or just suffered simple transverse or spiral fracture, it can automatically reduce when inserting the tibial intramedullary nail and no valgus or varus deformity of the distal fragment will occur. However, when isthmus is comminuted such as AO fracture type 42-B3 and type C, the fracture cannot automatically reduce during the insertion of the tibial intramedullary nail. Under this circumstance the isthmus lost the physical restraint effect on the placement of intramedullary nails and resulted in the eccentric nail for distal fragment with the sequelae of valgus or varus deformity. 2) For the fracture at the 
distal $1 / 3$ of the tibia, with the existence of the cavernous distal tibial metaphysis, the intramedullary nails could not achieve effective contact with the bone cavity. In other words, the cortical bone has no sufficient physical restraint effect on the tibial intramedullary nail during insertion and may result in possible deviations from the lateral or medial sides on the coronary plane, by which finally cause valgus or varus deformity of the distal fragment. 3) Currently, the intraoperative fluoroscopic imaging just provides local view of the tibia and hardly provides an entire view of the full-length tibia in a single film, hence the mild valgus or varus deformity emerged on the coronal plane cannot be detected.

\section{The feasibility to determine the occurrence of distal tibia fragment valgus or varus deformity intraoperatively}

For patients with existed knee joint deformity before injury (knee valgus or valgus), the included angle between the axis of the distal horizontal interlocking screw and the horizontal line of the tibiotalar articular surface in the AP X-ray film cannot be used to determine whether the distal tibia fragment combine valgus or varus deformity. Because for such case scenario there existed deviations in the entry point of the tibia intramedullary nail, and the standard entry point for the nails cannot be obtained as shown in Fig. 2. Which means that the intramedullary nail may not get into the center of the tibial bone marrow cavity in the coronal plane when it enters the proximal end of the medullary cavity.

During the surgery, the $\mathrm{C}$-arm fluoroscopy can easily obtain the AP images of the distal tibia. It is preferable to get standard AP films, by which the included angle between the axis of the distal horizontal interlocking screw and the horizontal line of the tibiotalar articular surface on the AP view represents the actual value, which is more accurate when correcting the valgus or varus deformity and adjusting the implant position. We preferred the fibular bisector line (overlap of the lateral border of the tibia bisecting the fibula head) as a reliable intraoperative fluoroscopic confirmation of appropriate rotation [11].

If an included angle emerged by the projections of the distal horizontal interlocking screw and the tibiotalar articular surface on the coronal plane, it is demonstrated that the valgus or varus deformity occurs at the distal fragment of the tibia. Meanwhile the fibula can be used as a reference. If the included angle points to the fibular side, the valgus deformity exists, and if the angle faces away from the fibula, then varus deformity exists. In this study, 33 patients with tibial comminuted fractures were evaluated by this method during the surgery, and the postoperative alignment and clinical outcomes were satisfactory.

The distal end of the tibial intramedullary nail is designed as two locking holes on the sagittal plane. With regard to the dynamic locking hole, to the best of our knowledge, there is currently no tibial intramedullary nail with a dynamic hole at the distal end. Oblique interlocking angulation may occur when the screw is locked through the dynamic hole by freehand technique, which means the interlocking screw is not perpendicular to the main nail. However, the oblique interlocking phenomenon would not occur when locking through the static locking hole. Therefore, the freehand locking technique does not create the bias to our method when it is applied to the tibial intramedullary nail through a static hole.

\section{The Significance Of Developing This Convenient Approach}


Previous studies [23-25] showed the application of a femoral distractor to maintain the tibial alignment. During the surgery, the traction pins are placed posterior to the central axis of the proximal tibial metaphysis and the distal end of the tibia. The perpendicular relationship between the intramedullary nail and the traction pins or the tibiotalar articular surface is used to verify the validity of fracture reduction. We have also tried to apply this method, however we found it has some limitations. Firstly, the inserted traction pin must be perpendicular to the tibial force line which is difficult to operate on the fractured tibia; secondly, the distractor is fixed with a single pin at the proximal and distal site which implies poor ability on anti-rotation and later reduction. Therefore, it is not practical to apply this method. In addition, some authors $[10,11]$ suggest that the guide wire of the intramedullary nail at the distal end must be centered before reaming so that central reaming can ensure that the distal fracture fragment can be well controlled after the insertion of intramedullary nails, and diminishing the probability of poor postoperative alignment or deformity. However, based on our clinical experience, eccentric placement of the intramedullary nail still occurs in some patients even the guide wire is in the center of cavity during reaming for the elderly patients with osteoporosis. This is likely resulted from insufficient cancellous bone mass of the distal tibia. Therefore, it has certain limitations for elderly patients with tibial fractures who combines osteoporosis. Of the 33 patients in our study, 5 patients over 60 years old with osteoporosis were estimated by our method. Postoperative full-length X-ray films demonstrated no valgus or varus deformity of the distal fragment. Hence we believe that this method is a simple and convenient approach to determine the occurrence of distal tibia fragment valgus or varus deformity intraoperatively and allow the surgeons to correct the angulations immediately without using additional tools.

\section{Conclusion}

In summary, in the treatment of tibial fractures with closed reduction and intramedullary nail fixation, the included angle between the distal horizontal interlocking screw and the tibiotalar articular surface on the AP X-ray film can be used to effectively determine the occurrence of distal tibia fragment valgus or varus deformity intraoperatively after the tibial intramedullary nail inserted through the standard entry point. However, this method requires at least one distal horizontal locking screw to be locked firstly. When valgus or varus deformity occurs, the screw has to be removed and then drilled and locked again after correction by poller screw. The procedure may cause certain damage to the distal tibia and further improvement is necessary.

\section{Declarations}

\section{Ethics approval and consent to participate}

This study was performed in accordance with the Declaration of Helsinki as revised in 2008 and was authorized by the Ethics Committee of Xi'an Honghui Hospital.

\section{Availability of data and materials}


The data and materials are available from the medical records department of Xi'an Honghui Hospital.

\section{Competing interests}

The authors declare that they have no competing interests

\section{Availability of data and materials}

The data and materials are available from the medical records department of Xi'an Honghui Hospital.

\section{Funding}

Not applicable

\section{Author's contributions}

YBX and TM Designed the study, modified and approved the final version of the manuscript. YL and JY wrote the first draft of the manuscript. ML CR QW ZL and KZ collected, analyzed and interpreted the data. All authors read and approved the final manuscript.

\section{References}

1. Cannada LK, Mir HR, Kottmeier SA. Clinical Faceoff: Suprapatellar Tibial Nailing for Tibia Fractures. Clin Orthop Relat Res.2020,478(6):1178-1182. doi:10.1097/corr.0000000000001254

2. Piątkowski K, Piekarczyk P, Kwiatkowski K, Przybycień M, Chwedczuk B .Comparison of different locking plate fixation methods in distal tibia fractures. Int Orthop.2015, 39 (11):2245-2251. doi:10.1007/s00264-015-2906-4

3. Richard RD, Kubiak E, Horwitz DS . Techniques for the surgical treatment of distal tibia fractures. Orthop Clin North Am. 2014,45 (3):295-312. doi:10.1016/j.ocl.2014.04.001

4. Zhang J, Ebraheim NA, Li M, He X, Liu J, Zhu L,et al . External Fixation Using a Locking Plate: A Reliable Way in Treating Distal Tibial Fractures. J Orthop Trauma.2014, 29 (11):454-458. doi:10.1097/bot.0000000000000377

5. Alho A, Benterud JG, Høgevold HE, Ekeland A, Strømsøe K . Comparison of functional bracing and locked intramedullary nailing in the treatment of displaced tibial shaft fractures. Clin Orthop Relat Res. 1992,(277):243-250

6. Court-Brown CM, Christie J, McQueen MM. Closed intramedullary tibial nailing. Its use in closed and type I open fractures. J Bone Joint Surg Br. 1990,72 (4):605-611

7. Webb LX, Bosse MJ, Castillo RC, MacKenzie EJ. Analysis of surgeon-controlled variables in the treatment of limb-threatening type-III open tibial diaphyseal fractures. J Bone Joint Surg Am.2007, 89 (5):923-928. doi:10.2106/jbjs.F.00776

8. Freedman EL, Johnson EE. Radiographic analysis of tibial fracture malalignment following intramedullary nailing. Clin Orthop Relat Res.1995, (315):25-33 
9. Gustilo RB, Merkow RL, Templeman D. The management of open fractures. J Bone Joint Surg Am.1990, 72 (2):299-304

10. Bong MR, Kummer FJ, Koval KJ, Egol KA . Intramedullary nailing of the lower extremity: biomechanics and biology. J Am Acad Orthop Surg.2007,15 (2):97-106. doi:10.5435/00124635200702000-00004

11. Stinner DJ, Mir H. Techniques for intramedullary nailing of proximal tibia fractures. Orthop Clin North Am.2014, 45 (1):33-45. doi:10.1016/j.ocl.2013.09.001

12. Paley D, Tetsworth K. Mechanical axis deviation of the lower limbs. Preoperative planning of multiapical frontal plane angular and bowing deformities of the femur and tibia. Clin Orthop Relat Res.1992, (280):65-71

13. Olerud C, Molander H.A scoring scale for symptom evaluation after ankle fracture. Arch Orthop Trauma Surg. 1984,103(3):190-194. doi:10.1007/bf00435553

14. Ristiniemi J, Flinkkilä T, Hyvönen P, Lakovaara M, Pakarinen H, Biancari F, et al. Two-ring hybrid external fixation of distal tibial fractures: a review of 47 cases. J Trauma.2007, 62 (1):174-183. doi:10.1097/01.ta.0000215424.00039.3b

15. Avilucea FR, Sathiyakumar V, Greenberg SE, Ghiam M, Thakore RV, Francois E, et al. Open distal tibial shaft fractures: a retrospective comparison of medial plate versus nail fixation. Eur $\mathrm{J}$ Trauma Emerg Surg.2016, 42 (1):101-106. doi:10.1007/s00068-015-0519-7

16. Li Y, Jiang X, Guo Q, Zhu L, Ye T, Chen A.Treatment of distal tibial shaft fractures by three different surgical methods: a randomized, prospective study. Int Orthop.2014, 38 (6):1261-1267. doi:10.1007/s00264-014-2294-1

17. Zhou Y, Wang Y, Liu L, Zhou Z, Cao X. Locking compression plate as an external fixator in the treatment of closed distal tibial fractures. Int Orthop.2015, 39 (11):2227-2237. doi:10.1007/s00264015-2903-7

18. Jöstl J, Tiefenböck TM, Hofbauer M, Winnisch M, Lang N, Hajdu S,et al. Distal tibial fractures: evaluation of different fixation techniques. Wien Klin Wochenschr .2017,129 (5-6):164-168. doi:10.1007/s00508-015-0730-x

19. Ricci WM, O'Boyle M, Borrelli J, Bellabarba C, Sanders R. Fractures of the proximal third of the tibial shaft treated with intramedullary nails and blocking screws. J Orthop Trauma .2001,15 (4):264-270. doi:10.1097/00005131-200105000-00005

20. Lefaivre KA, Guy P, Chan H, Blachut PA.Long-term follow-up of tibial shaft fractures treated with intramedullary nailing. J Orthop Trauma.2008, 22 (8):525-529. doi:10.1097/BOT.0b013e318180e646

21. Obremskey WT, Medina M. Comparison of intramedullary nailing of distal third tibial shaft fractures: before and after traumatologists. Orthopedics.2004, 27 (11):1180-1184

22. Puno RM, Vaughan JJ, Stetten ML, Johnson JR. Long-term effects of tibial angular malunion on the knee and ankle joints. J Orthop Trauma .1991,5 (3):247-254. doi:10.1097/00005131-19910900000001 
23. Park H, Ryu KJ, Kim HW, Hwang JH, Han JW, Lee DH. Is there an Increase in Valgus Deviation in Tibial Distraction Using the Lengthening Over Nail Technique? Clin Orthop Relat Res.2016, 474 (5):1283-1291. doi:10.1007/s11999-016-4712-8

24. Moed BR, Watson JT. Intramedullary nailing of the tibia without a fracture table: the transfixion pin distractor technique. J Orthop Trauma.1994, 8 (3):195-202. doi:10.1097/00005131-19940600000003

25. Baumgaertel F, Dahlen C, Stiletto R, Gotzen L. Technique of using the AO-femoral distractor for femoral intramedullary nailing. J Orthop Trauma.1994, 8 (4):315-321. doi:10.1097/00005131199408000-00007

\section{Figures}




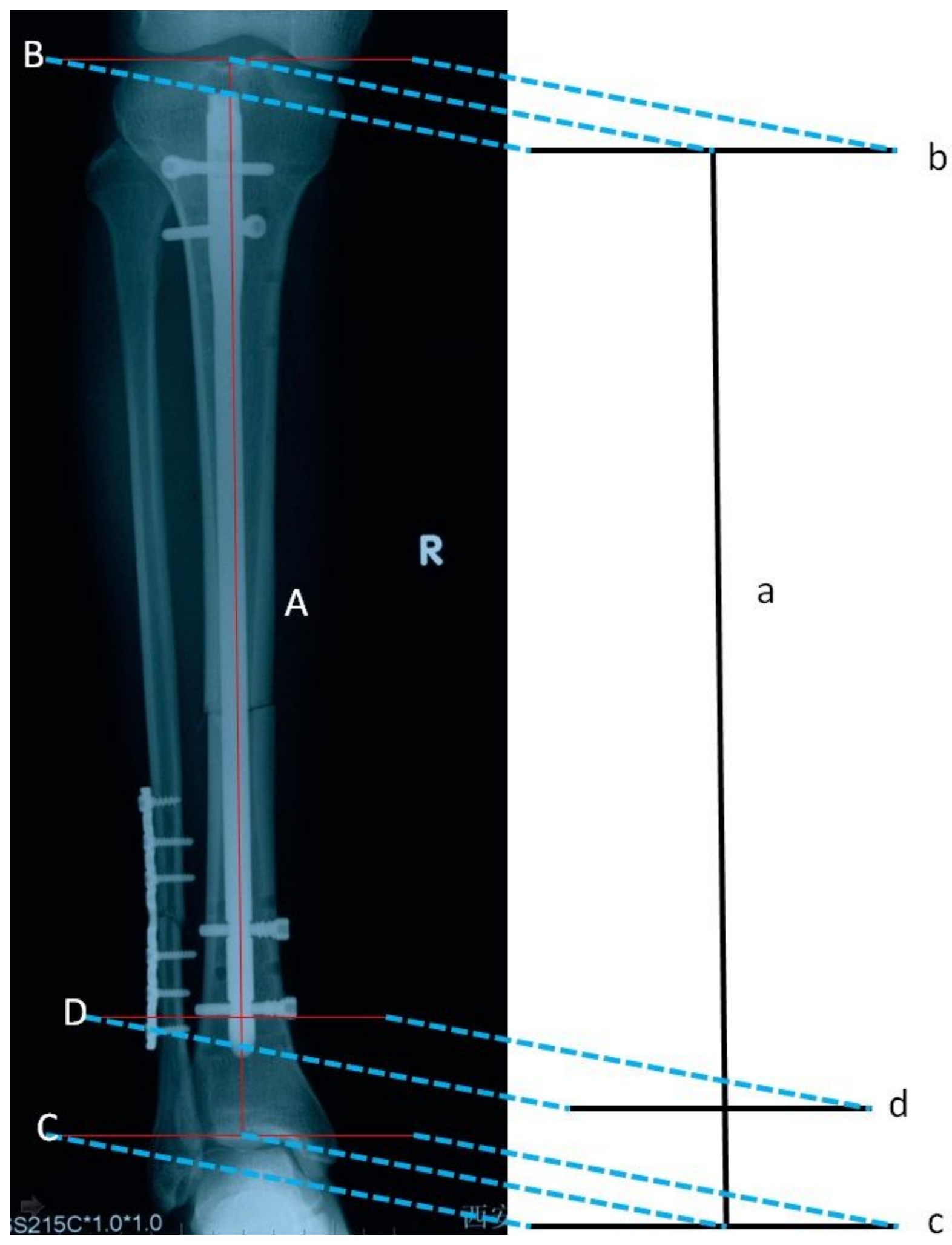

\section{Figure 1}

General view of the projection line on the coronal plane. The tibial intramedullary nail projection line (line a), the knee joint surface projection line (line b), the tibiotalar joint surface projection line (line $c$ ) and the axis of distal horizontal interlocking screw projection line (line d). 


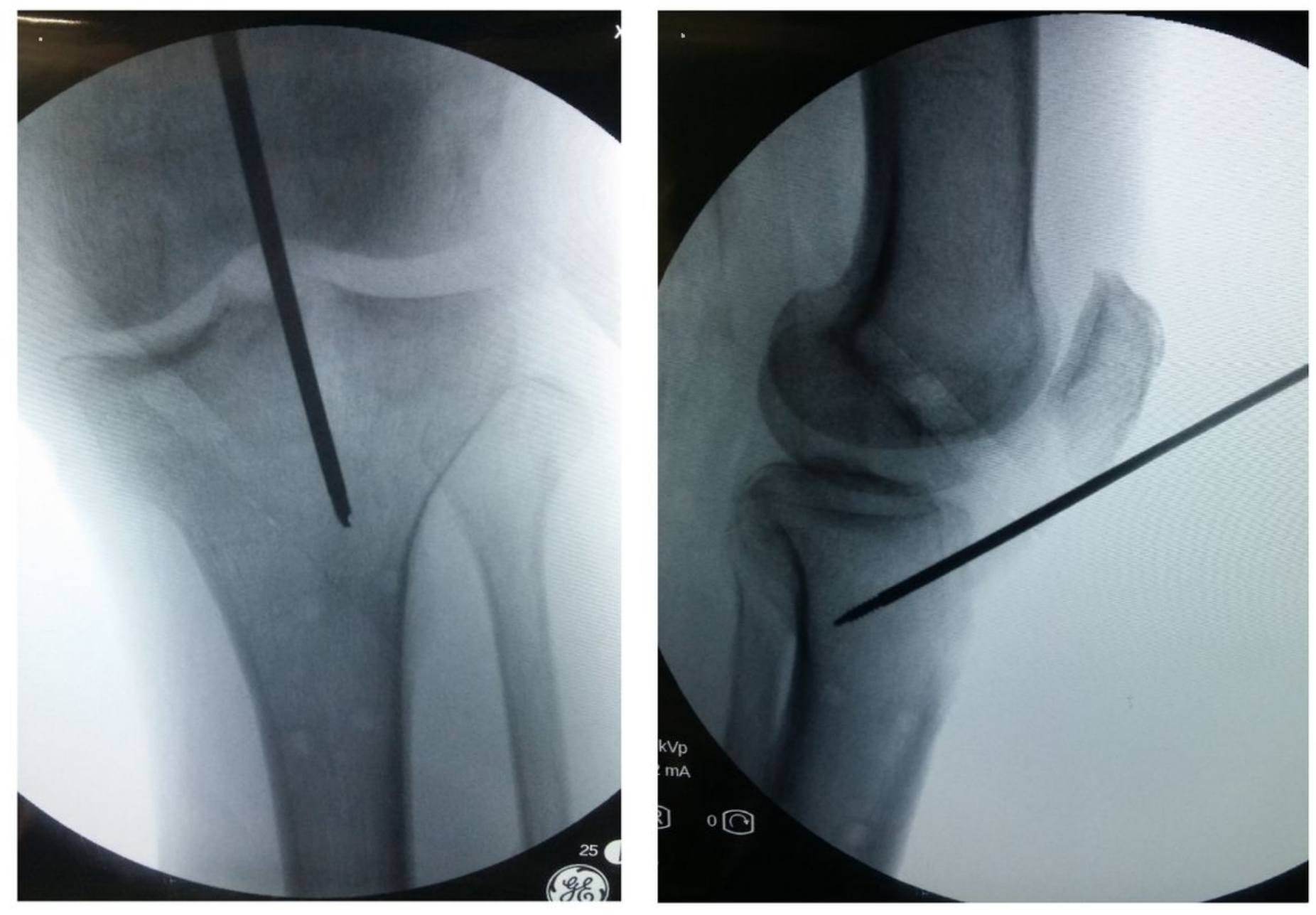

Figure 2

The ideal starting point of the guide wire on the AP and lateral views intraoperatively. 

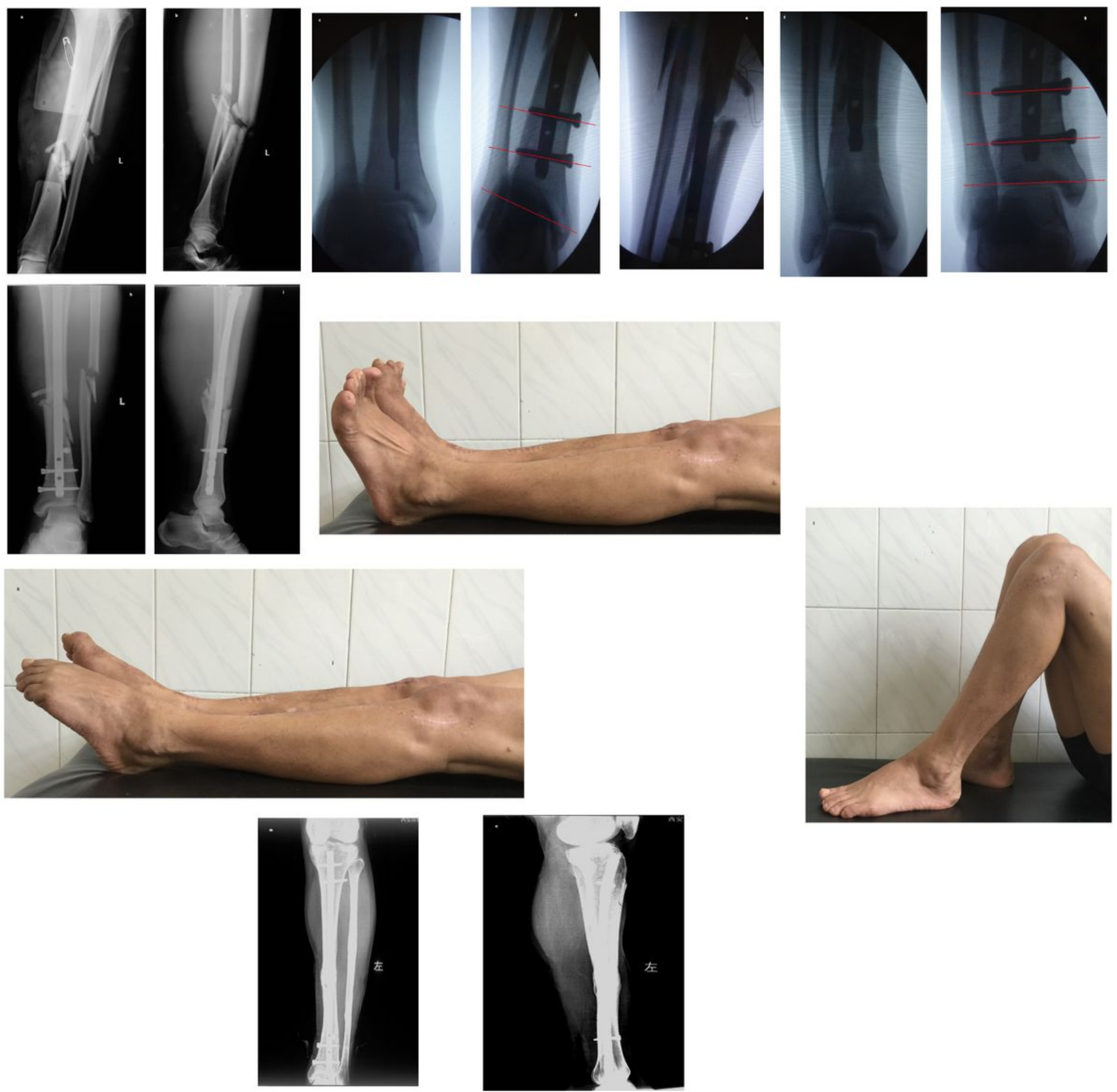

\section{Figure 3}

A-B: The patient was a 21-year-old male who experienced a road traffic accident causing left calf pain and limited activity. The $\mathrm{AP}(\mathrm{A})$ and lateral(B) radiographic results demonstrated a left tibia and fibula fracture (42-C3) . C: The insertion of the finger reduction device through the guide wire. D: The insertion of the nail after gradual reaming and the distal interlocking screws were locked by free hand procedure. An included angle emerged between line $\mathrm{c}$ and line $\mathrm{d}$ which pointed to the fibula. $\mathrm{E}$ : The AP view of the distal fragment demonstrated a valgus deformity. F: Reinsertion of the nail after application of the poller screw technique for the distal tibial fragment. G: The parallel relationship between line $\mathrm{c}$ and line $\mathrm{d}$ indicated the 
correction of the valgus deformity intraoperatively. $\mathrm{H}-\mathrm{I}$ : $\mathrm{AP}(\mathrm{H})$ and lateral $(\mathrm{I}) \mathrm{X}$-ray films 2 days after surgery demonstrated the satisfactory reduction, alignment and implants location. J-L: The range of motion for the knee and ankle joint at one year of follow up. M-N: The AP(M) and lateral(N) radiographs at one year of follow up. 